\title{
Incidentally Found Transient and Asymptomatic ST-Elevation: A Diagnostic Dilemma
}

\author{
Thomas Matteazzi ${ }^{\mathrm{a}}$, Nicola Martinelli ${ }^{\mathrm{a}}$, \\ Oliviero Olivieri ${ }^{\mathrm{a}}$, Domenico Tavella ${ }^{\mathrm{b}}$, Domenico Girelli ${ }^{\mathrm{a}, \mathrm{c}}$
}

\begin{abstract}
Acute coronary syndromes (ACSs) are among the most frequent causes of hospitalization worldwide. The diagnosis of ACS is based on three main criteria: 1) chest pain; 2) electrocardiographic changes; 3 ) changes in markers of myocardial cytolysis. However, one or more of these features are not infrequently missing or confounding, making the diagnosis difficult. An example of this condition is the so-called "silent ischemia". In this article we report a case of a 70 -year-old man in whom transient and asymptomatic ST-elevation was incidentally recorded during hospitalization. The management of this "out of guidelines" case is discussed in light of the increasing epidemic of silent myocardial ischemia.
\end{abstract}

Keywords: Acute coronary syndromes; Transient ischemic ST-elevation; Asymptomatic ST-elevation; Silent ischemia; Unrecognized myocardial infarction; Silent coronary artery disease

\section{Introduction}

Acute coronary syndromes (ACSs) are leading causes of death worldwide, and among the commonest medical emergencies encountered in daily clinical practice. The diagnosis of ACS is classically based on three main criteria: 1) chest pain with typical precordial distribution; 2) electrocardiographic (ECG) changes compatible with ischemia; and 3) characteristic pattern of changes in biomarkers of myocardial cytolysis, like troponins I or T (TnI, TnT) [1]. Nevertheless,

\footnotetext{
Manuscript accepted for publication July 4, 2014

${ }^{\mathrm{a} D e p a r t m e n t ~ o f ~ M e d i c i n e, ~ S e c t i o n ~ o f ~ I n t e r n a l ~ M e d i c i n e, ~ U n i v e r s i t y ~ o f ~}$ Verona, Italy

${ }^{\mathrm{b}}$ Unit of Cardiology, Azienda Ospedaliera Universitaria Integrata of Verona, Italy

${ }^{\mathrm{c}}$ Corresponding Author: Domenico Girelli, Department of Medicine, Section of Internal Medicine, University of Verona, Italy.

Email: domenico.girelli@univr.it
}

doi: http://dx.doi.org/10.14740/jmc1842w often one or more of these features are missing or confounding, making it difficult to decide whether or not a specific treatment is needed in a given patient. An example of such challenging conditions is the so-called "silent ischemia" [2], i.e. the presence of ECG changes apparently typical of myocardial ischemia (like ST deviations or T wave inversions), occurring in the absence of any clinical symptom or sign. This is particularly true when ST-elevation is recorded, and this change is commonly perceived as the most potentially dangerous and life-threatening. On this occasion, physicians have to consider the diagnosis of non-ischemic ST-elevation (NISTE) [3, 4], which can occur in a variety of clinical conditions. The clinical decision may be even more difficult if ST-elevation is rapidly transient, a condition that may be falsely reassuring. Indeed, missing the diagnosis of transient ischemic ST-elevation (TISTE) [5] may lead to dramatic consequences. Here we report a rather unique case of an elderly man in whom transient and asymptomatic ST-elevation was incidentally recorded during hospitalization because of another cause. The management of this "out of guidelines" case is discussed in light of the increasing epidemic of silent myocardial ischemia [2].

\section{Case Report}

A 70-year-old male was referred to the emergency room (ER) of our hospital because of fatigue, worsening of renal function (serum creatinine $318.6 \mu \mathrm{mol} / \mathrm{L}$, eGFR $24.71 \mathrm{~mL} /$ $\mathrm{min})$ and hypokalemia $(2.8 \mathrm{mmol} / \mathrm{L})$. On arrival, blood pressure was 190/90 mm Hg, heart rate was 68 beats per minute, and temperature was normal. A (3/6) systolic heart murmur was felt in the aortic focus, while chest auscultation revealed subtle basal crackles. There was no peripheral edema, but signs of severe peripheral artery disease (PAD) were present, i.e. an ulcer on the heel of the right foot with signs of superimposed infection and a dry necrosis of the second toe of the left foot. For this reason, the patient had been regularly followed by colleagues of the local vascular rehabilitation unit in the previous months, after consultation by a vascular surgeon that had excluded any possibility of intervention. The ECG recorded in the ER showed atrial fibrillation, in line 

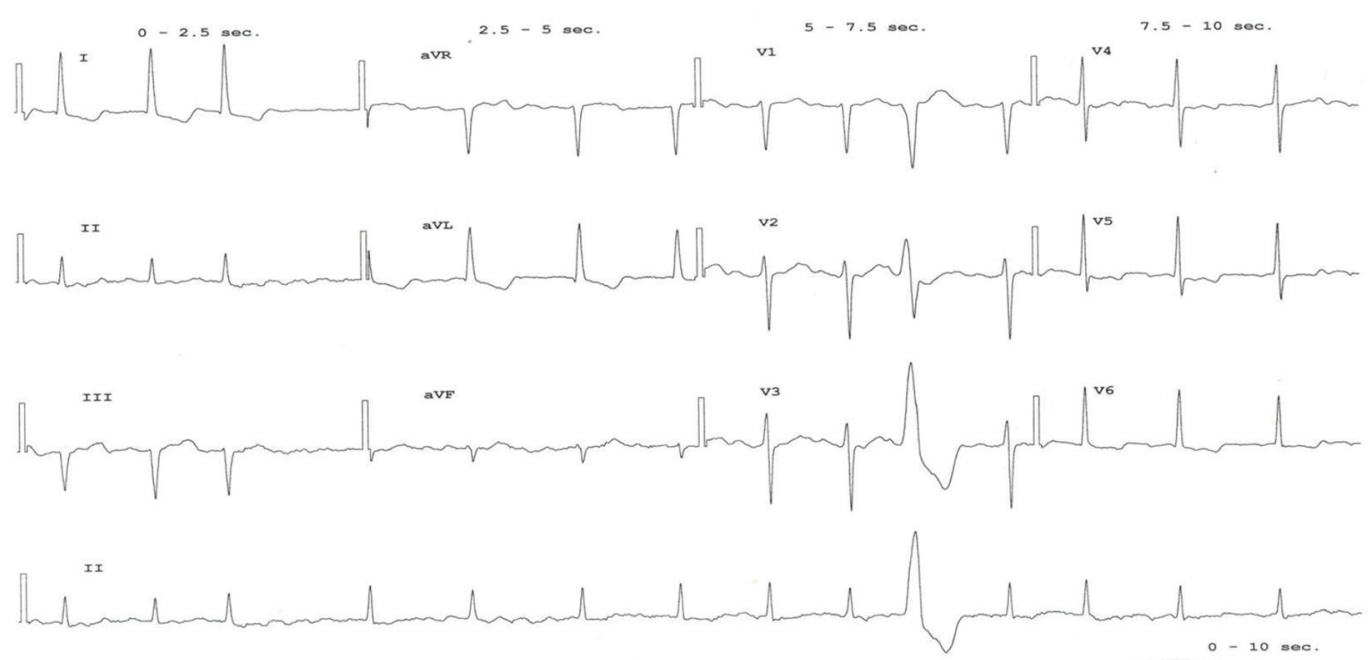

Figure 1. The ECG recorded on admission, showing the known permanent atrial fibrillation of the patient, without signs of cardiac ischemia.

with patient's history, without any ischemic changes (Fig. 1).

History revealed permanent atrial fibrillation, poorly controlled type 2 diabetes mellitus, hypertension, chronic kidney disease (stage III, with mean creatinine level around $278.1 \mu \mathrm{mol} / \mathrm{L}$ ), previous cerebral stroke, recurrent episodes of acute cardiac decompensation, peripheral atherosclerosis with documented carotid and femoral stenosis, and chronic gastritis. No previous episodes of ACS were reported. The patient assumed warfarin, furosemide $125 \mathrm{mg}$ daily, canrenone $25 \mathrm{mg}$ daily, lansoprazole $30 \mathrm{mg}$ daily, doxasozin 4 $\mathrm{mg}$ daily, lorazepam $1 \mathrm{mg}$ daily, allopurinol $100 \mathrm{mg}$ daily, aspirin $100 \mathrm{mg}$ daily, rosuvastatin $20 \mathrm{mg}$ daily, amiodarone
$200 \mathrm{mg}$ daily, clopidogrel $75 \mathrm{mg}$ daily, regular insulin $16 \mathrm{IU}$ three times a day, glargine insulin 22 IU daily, nitroglycerin (transdermal patches) $5 \mathrm{mg}$ daily and fentanil (transdermal patches) $25 \mathrm{mg}$ every $72 \mathrm{~h}$. After hospital admission, the patient was treated with antibiotics (levofloxacin and ceftazidime) and cautious intravenous hydration with amelioration of renal function, and there were no signs of heart failure. Few days after admission, during the morning visit the patient reported himself as ameliorated, asking for returning home. On physical examination a regular pulse was found, which prompted ordering a second ECG just to check for a spontaneous restoration of sinus rhythm. Unexpectedly, the

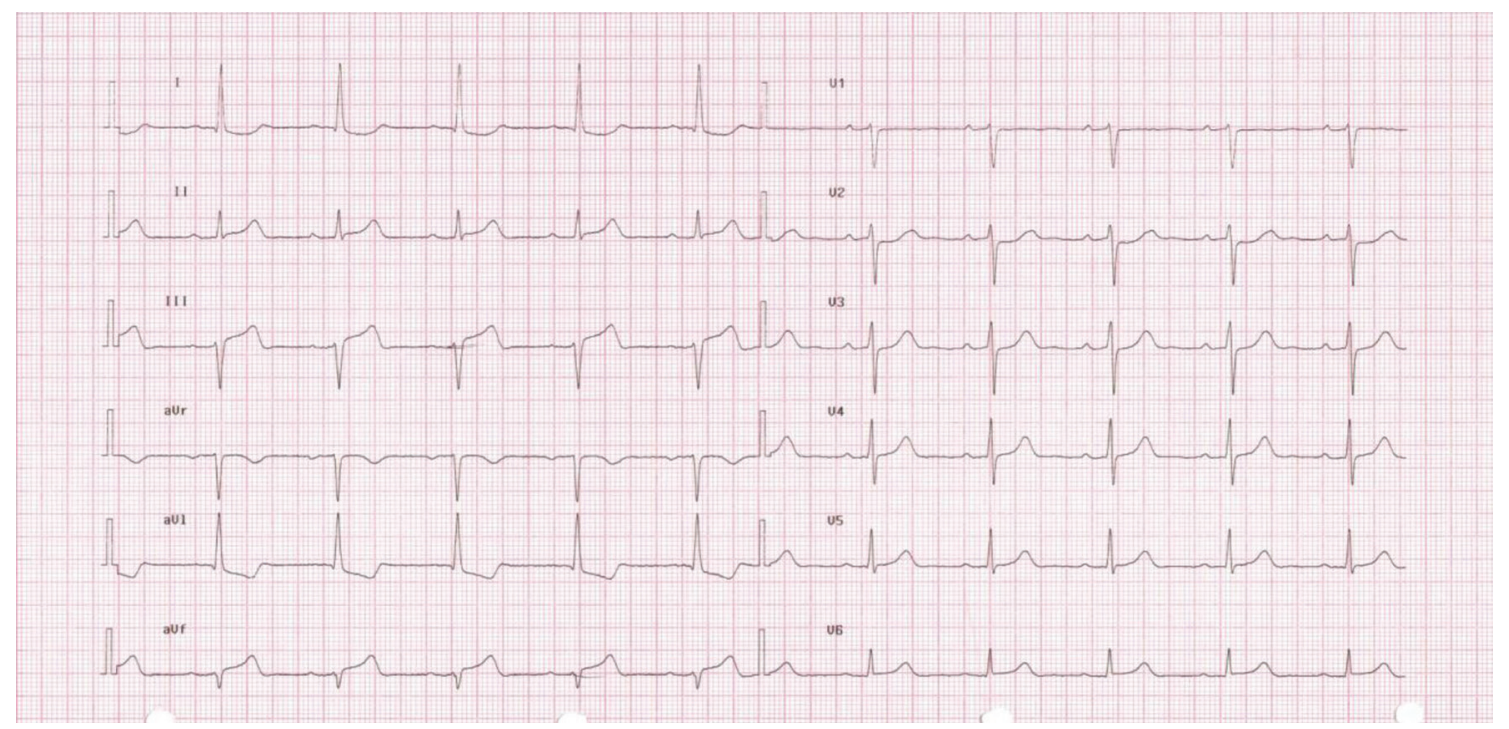

Figure 2. ECG showing ST-elevation in DII, DIII and aVF (7:35 am). 


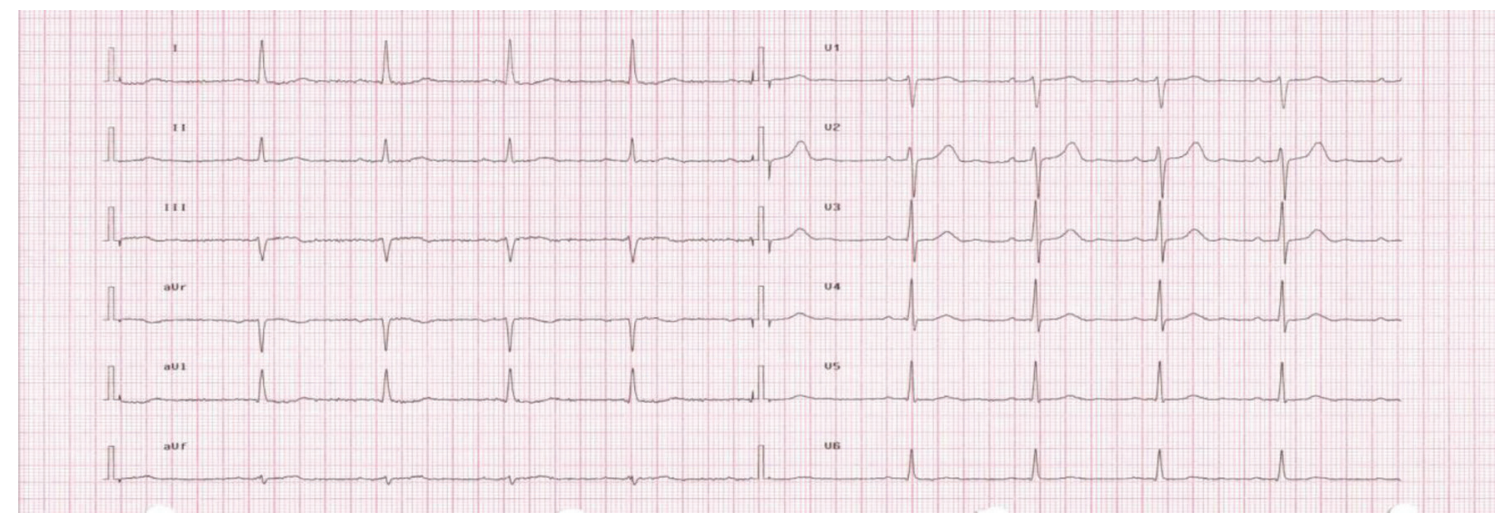

Figure 3. ECG showing the resolution of the ST-elevation performed after $1 \mathrm{~h}(8: 38 \mathrm{am})$.

ECG found an ST-elevation on leads DII, DIII and aVF (Fig. 2 ), suggestive of subepicardial inferior ischemia. The patient was hemodynamically stable and free of signs of heart failure. A bedside ECG did not show any wall motion abnormalities, particularly in the inferior wall. Serum markers of myocardial injury also did not show a significant increase. Indeed, initial high-sensitive troponin I (hs-TpnI) level was altered $(0.139 \mu \mathrm{g} / \mathrm{L}$; normal value $<0.01 \mu \mathrm{g} / \mathrm{L})$ but this could be attributed to renal disease, and creatine kinase MB fraction was normal $(3.3 \mu \mathrm{g} / \mathrm{L})$. A second hs-TpnI checked $3 \mathrm{~h}$ after a stable pattern $(0.134 \mu \mathrm{g} / \mathrm{L})$ is confirmed. Of note, a second ECG performed about $60 \mathrm{~min}$ after first recording documented a complete resolution of the ST-elevation (Fig. $3)$.

The case was rapidly discussed with a cardiology consultant. Notwithstanding some apparently reassuring findings, like the lack of symptoms, hemodynamic stability, negative ECG and laboratory studies, unstable coronary artery disease (CAD) was considered a likely diagnosis. The pa-

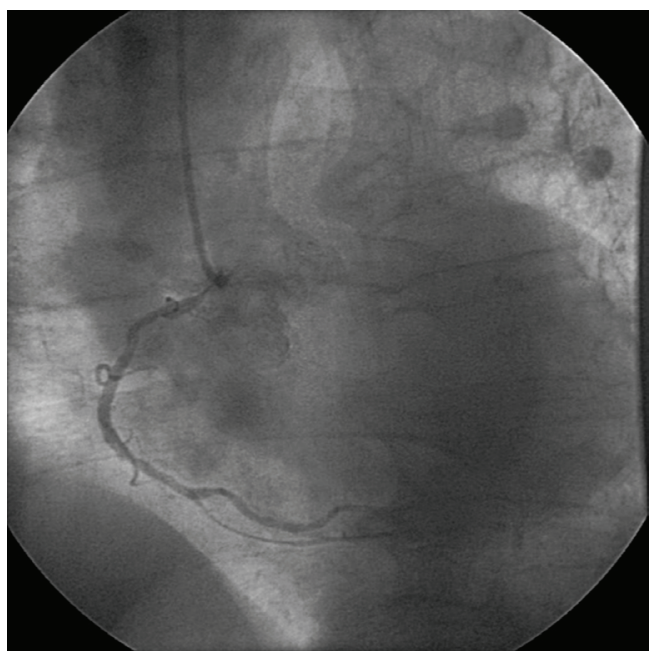

Figure 4. Coronary angiography showing sub-occlusive stenosis at the ostium of the right coronary artery. tient was addressed to the cardiac catheterization laboratory, and a coronary angiography was performed. The angiography documented a severe CAD with a sub-occlusive stenosis $(70-80 \%)$ at the ostium of right coronary artery, and a double proximal stenosis (60-70\%) of the same vessel (Fig. 4).

Effective revascularization of the right coronary artery was performed by applying a drug eluting stent (DES), without any immediate and late complications. Serial ECGs were unremarkable for further ischemic changes and showed persistent restoration of sinus rhythm. Similarly, renal function was stable (serum creatinine at discharge $249 \mu \mathrm{mol} / \mathrm{L}$ ). Few days later the patient was discharged with addition of dual anti-platelet therapy (aspirin and clopidogrel) and of amiodarone. He was included in a program of serial ambulatory medication by the vascular surgery unit.

\section{Discussion}

This rather unique and challenging case underscores the difficulties of diagnosing ACS, especially when patients do not present with the classic triad of suggestive symptoms, dynamic ECG changes, and typical alterations of biomarkers of myocyte injury [1]. A prompt diagnosis and treatment of ACS are essential to avoid short-term and long-lasting adverse effects [6]. Notwithstanding the incidental finding of ECG changes and the absence of any ACS symptom, our patient could be considered as high-risk subject because of the presence of several well-known risk factors for CAD, i.e. PAD [7], diabetes mellitus and chronic kidney disease [8]. Moreover, he had an infected diabetic foot ulcer, which has been associated to an increased risk of fatal myocardial infarction [9]. Of note, there is increasing evidence that acute infections can trigger ACS in patients with underlying CAD through multiple mechanisms including systemic inflammation, induction of a procoagulant status and hypoxia/ increased metabolic demand [10]. All together, these risk conditions prompted to consider the incidentally observed 
ECG ST-elevation as the result of a true myocardial ischemia, even if it was transient (lasting less than $1 \mathrm{~h}$ ), and less than $2 \mathrm{~mm}$ in width. Indeed, recent guidelines require that ST-elevation has to be not lower than $2 \mathrm{~mm}$ to be classified as ACS-STEMI [11]. The differential diagnosis in this case included several patho-physiological conditions possibly associated to a NISTE [4]. Nfor et al (2012) studied 54 patients with angiographically proven NISTE, i.e. subjects who received emergency cardiac catheterization because of suspected STEMI in whom no culprit lesion could be demonstrated. The authors identified four major features significantly associated with NISTE, including absence of chest pain (risk score 3), absence of reciprocal ST changes on ECG (risk score 3), symptoms duration $>6 \mathrm{~h}$ (risk score 2 ) and a reduced burden $(<3)$ of classical risk factors for CAD as compared to patients with true STEMI (risk score 2). The most frequent post-hoc diagnosis of patients with NISTE included early repolarization (14.8\%), myocarditis/ pericarditis $(14.8 \%)$, stress-induced (tako-tsubo) cardiomyopathy (11.1\%) and coronary spasm (9.3\%). Of note, the application of the score proposed by the authors to our patient would have result on point 6 , i.e. a relatively high probability $(80 \%)$ of not identifying a culprit coronary lesion [3]. Besides the two factors mentioned above, i.e. absence of both chest pain and reciprocal ST changes, our patient had indeed two other potentially reassuring conditions, like the lack of changes in hs-TpnI and of abnormal ECG findings. However, our clinical judgment was mostly influenced by the perception of facing a patient at very high risk of CAD. This illustrates the need to improve the available algorithms for differentiating NISTE from TISTE/STEMI in the emergency setting. The study by Nfor and colleagues included a relatively small number of patients, and the score they proposed for NISTE/STEMI differentiation has to be considered with great caution. Further studies are needed in larger number of patients. The case here described is peculiar even because the TISTE was detected incidentally during an ECG recorded for a reason other than suspected ACS, in a patient completely asymptomatic for typical or atypical ACS manifestations. Thus, it is nearly impossible to compare this patient with any published series. A condition that may mimic what we observed is represented by asymptomatic ST-elevation changes recorded during $24 \mathrm{~h}$ Holter ECG. In the IMMEDIATE AIM Study, Drew and colleagues described five patients who were discharged from the ER with a presumed non-cardiac diagnosis, and shortly thereafter showed silent ST alterations at an ECG Holter recorded by study protocol. During a 1-year follow-up two of these patients with "missed" transient ST alterations died, and a third had an acute myocardial infarction (AMI) [5]. This underscores the negative prognostic impact of transient silent ECG changes if they are left untreated. Indeed, silent ischemia/infarction is growing phenomenon in clinical practice. In epidemiological studies, the relative proportion of the so-called unrecognized myocardial infarction (UMI) among total AMI ranged from a minimum of $22 \%$ in the Cardiovascular Health Study (2000) to a maximum of 44\% in the Bronx Aging Study (1990) [2]. A recent study based on cardiac magnetic resonance in 936 community-dwelling elderly subjects (aged 67 - 93 years), the prevalence of UMI was nearly two-fold higher than that of recognized myocardial infarction (RMI), i.e. 17\% versus $9.7 \%$, respectively. Of note, during a median follow-up of 6.4 years UMI showed a worse prognosis than RMI, likely because UMI patients did not receive the same treatments as RMI patients [12]. Hypertension, elderly, diabetes mellitus, female gender and chronic kidney disease have been identified as the clinical conditions with the highest association with silent ischemia $[2,13]$. Of note, when compared with symptomatic ischemia, silent ischemia is more likely to occur during periods of physical inactivity, i.e. during sleep or bed rest (as in our patient), as well as at a lower heart rate [14].

\section{Conclusion}

Beyond the traditional view, ACS is characterized by a marked clinical heterogeneity, including a growing number of cases without chest pain. This "epidemic" of silent CAD is likely to further increase due to population aging, and the consequent multi-morbidity. In parallel, technological advances allow increased diagnosis of unrecognized ACS. The peculiar patient here described illustrates a paradigm of a diagnostic dilemma in which currently available guidelines are poorly useful, as well as the urgent need of further studies in the field.

\section{Abbreviations}

ACS: acute coronary syndrome; STEMI: ST-elevation myocardial infarction; NISTE: non-ischemic ST-elevation; TISTE: transient ischemic ST-elevation; CAD: coronary artery disease; PAD: peripheral artery disease; AMI: acute myocardial infarction; UMI: unrecognized myocardial infarction

\section{References}

1. Kumar A, Cannon CP. Acute coronary syndromes: diagnosis and management, part I. Mayo Clin Proc. 2009;84(10):917-938.

2. Sheifer SE, Manolio TA, Gersh BJ. Unrecognized myocardial infarction. Ann Intern Med. 2001;135(9):801811.

3. Nfor T, Kostopoulos L, Hashim H, Jan MF, Gupta A, Bajwa T, Allaqaband S. Identifying false-positive ST-elevation myocardial infarction in emergency department 
patients. J Emerg Med. 2012;43(4):561-567.

4. Wang K, Asinger RW, Marriott HJ. ST-segment elevation in conditions other than acute myocardial infarction. N Engl J Med. 2003;349(22):2128-2135.

5. Drew BJ, Schindler DM, Zegre JK, Fleischmann KE, Lux RL. Estimated body surface potential maps in emergency department patients with unrecognized transient myocardial ischemia. J Electrocardiol. 2007;40(6 Suppl):S15-20.

6. De Luca G, Suryapranata H, Zijlstra F, van 't Hof AW, Hoorntje JC, Gosselink AT, Dambrink JH, et al. Symptom-onset-to-balloon time and mortality in patients with acute myocardial infarction treated by primary angioplasty. J Am Coll Cardiol. 2003;42(6):991-997.

7. Aronow WS, Ahn C. Prevalence of coexistence of coronary artery disease, peripheral arterial disease, and atherothrombotic brain infarction in men and women $>$ or $=$ 62 years of age. Am J Cardiol. 1994;74(1):64-65.

8. Go AS, Chertow GM, Fan D, McCulloch CE, Hsu CY. Chronic kidney disease and the risks of death, cardiovascular events, and hospitalization. N Engl J Med. 2004;351(13):1296-1305.

9. Brownrigg JR, Davey J, Holt PJ, Davis WA, Thompson
MM, Ray KK, Hinchliffe RJ. The association of ulceration of the foot with cardiovascular and all-cause mortality in patients with diabetes: a meta-analysis. Diabetologia. 2012;55(11):2906-2912.

10. Corrales-Medina VF, Madjid M, Musher DM. Role of acute infection in triggering acute coronary syndromes. Lancet Infect Dis. 2010;10(2):83-92.

11. Thygesen K, Alpert JS, White HD. Universal definition of myocardial infarction. J Am Coll Cardiol. 2007;50(22):2173-2195.

12. Schelbert EB, Cao JJ, Sigurdsson S, Aspelund T, Kellman P, Aletras AH, Dyke CK, et al. Prevalence and prognosis of unrecognized myocardial infarction determined by cardiac magnetic resonance in older adults. JAMA. 2012;308(9):890-896.

13. Lee MG, Jeong MH, Lee KH, Park KH, Sim DS, Yoon HJ, Yoon NS, et al. Comparison of chronic kidney disease and risk for presenting with painless versus nonpainless acute myocardial infarction. Am J Cardiol. 2012;110(6):790-794.

14. Cohn PF. Silent myocardial ischemia as a manifestation of asymptomatic coronary artery disease: what is appropriate therapy? Am J Cardiol. 1985;56(6):28D-34D. 